\title{
ANÁLISE ESTATÍSTICA DE PARÂMETROS ELÉTRICOS EM USINAS SUCROALCOLEIRAS DO CENTRO-OESTE PAULISTA
}

\author{
Maria Cristina Rodrigues Halmeman', Luís Roberto A. Gabriel Filho², Odivaldo José \\ Seraphim ${ }^{3}$, Luiz Antonio Perea ${ }^{4}$ e Radames Juliano Halmeman ${ }^{5}$
}

RESUMO: A indústria da cana-de-açúcar tornou-se um importante colaborador na geração de energia elétrica no Brasil. Atualmente há 434 usinas de açúcar e etanol em operação no país e deste total 23\% exportam energia elétrica para o Sistema Integrado Nacional (SIN). No estado de São Paulo há 182 usinas e 30\% delas exportam energia para o sistema. O objetivo deste trabalho é comparar parâmetros de eficiência elétrica no setor sucroalcooleiro. Para o estudo, foram escolhidas três usinas com grande potencial de produção e exportação de açúcar e etanol da região centro-oeste do estado de São Paulo. Foram utilizados, para aferição de parâmetros elétricos, cinco analisadores de energia LANDYS+GYR SAGA. As variáveis analisadas foram a energia consumida (C) e Fator de Potência (FP). Para a análise estatística, adotou-se o delineamento de blocos casualizados, em arranjo fatorial $3 \times 5$, composto das três empresas sucroalcoleiras e cinco setores de consumo energético sendo: recepção (1), moagem (2), caldeiras (3), atividades auxiliares/tratamento de caldo (4) e destilaria (5) totalizando 15 tratamentos, sendo cada grupo composto por 192 repetições (48 horas $\times 4$ aferições por hora). Conclui-se que não há uma preocupação por parte das usinas em corrigir o (FP) e atingir o valor de 0,92, que é considerado o fator de potência ideal, visto que as usinas, por gerarem sua própria energia, não são penalizadas pelas concessionárias por apresentarem valores abaixo do recomendado. Em relação à energia consumida todos os setores apresentam diferenças significativas. Na comparação setor a setor, a usina denominada USB apresentou diferenças não significativas nos setores 1 e 3 e a usina USC nos setores 1 e 4 . Considerando as unidades produtoras do setor sucroalcooleiro e que vendem energia elétrica esse tipo de avaliação é essencial realizar essa analise, uma vez que os setores analisados são os mais importantes na produção do açúcar e etanol, e analisar e acompanhar esses parâmetros de uso e consumo de energia pode propiciar maior oferta de energia a ser comercializada.

PALAVRAS-CHAVE: analisadores de energia, fator de potência, energia consumida.

\section{STATISTICS ANALYSES OF THE ELECTRIC PARAMETERS IN SUGAR CANE PLANTS AT CENTRAL WEST OF SAO PAULO STATE}

\begin{abstract}
The industry of sugar cane has become an important contributor to the generation of electricity in Brazil. Currently there are 434 sugar and ethanol plants operating in the country, $23 \%$ of the total export electricity to the National Integrated System (SIN), the state of São Paulo has 182 plants and 30\% of them export energy to the SIN. The objective of this study is to compare parameters of electrical efficiency in the sugar and alcohol industry. For the study, three plants localized in the midwest region of Sao Paulo state with great potential for production and exporting bioenergy were chosen. Five energy analyzers LANDYS + GYR SAGA were used for measure the electrical parameters. The variables studied were energy consumption (C) and power factor (PF). For the statistical analysis it was adopteda randomized block design in a factorial $3 \times 5$ composed of three companies and five sectors of energy consumption,in which: reception(1), milling (2), boiler (3), supporting activities / juice treatment (4), and distillation (5), totaling 15 treatments. Each group comprised 192 repetitions (48 hours $\times 4$ measurements per hour). It was concluded that there is no interest for the plans to fix the FP and reach a value 0.92 , which is considered the ideal power factor.This,because the plants generate their own energy and are not penalized. Regarding the energy consumed, all sectors had significant differences. When comparingsector to sector, the plant called USB showed no significant differences in sectors 1 and 3 , and the plant USC, in sectors 1 and 4. Considering the production units of this sector and selling power this type of evaluation is essential to perform this analysis, since the analyzed sectors are most important in the production of sugar and ethanol, and analyze and monitor these parameters, use and consumption energy can provide a greater supply of energy to be commercialized.
\end{abstract}

KEYWORDS: power Analyzers, power factor, energy consumed.

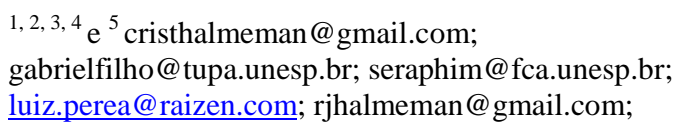




\section{INTRODUÇÃO}

A indústria da cana-de-açúcar tornou-se um importante colaborador na geração de energia elétrica no Brasil. Além de produtos como açúcar e etanol, o setor de produção e venda de energia elétrica contribuiu significativamente com a produção de 148.020 milhões de toneladas de bagaço de cana em 2009, responsável pela produção de 14.057 GWh de energia elétrica (EMPRESA DE PESQUISA ENERGÉTICA (BRASIL), 2010).

Atualmente há 434 usinas de açúcar e etanol em operação no país, deste total 23\% exportam energia para o Sistema Integrado Nacional (SIN). No estado de São Paulo, do total de 182 usinas, 30\% exportam energia para o sistema (UNIÃO DA INDÚSTRIA DE CANADE-AÇUCAR, 2011).

Conforme Anselmi (2009), nos últimos 10 anos aumentou a demanda por energia elétrica no país e consequentemente o interesse pela venda e geração de bioeletricidade. Devido a esse interesse, aconteceram diversas modificações nos processos de produção como a eletrificação de picadores e moendas, substituição de caldeiras e turbinas, melhorias para redução do consumo de vapor no processo, entre outras, o objetivo é aumentar os excedentes de eletricidade que podem ser comercializados.

A complexidade dos sistemas em usinas de açúcar e etanol, a variedade de equipamentos, as configurações de equipamentos produtores e consumidores de energia entre outros fatores tornam a tarefa de análise da eficiência energética complexa.

Conforme Filgueira et al. (2006) o uso de medidores de energia servem como base para o combate ao desperdício de energia e devem possuir, no mínimo, os seguintes recursos: memória de massa para armazenamento de dados; possibilidade de medição em sistemas monofásicos, bifásicos ou trifásicos; controle de demanda e fator de potência; ligação direta a sinais de tensão e corrente provenientes dos circuitos secundários dos transformadores de corrente (tc's) e dos transformadores de potência (tp's); monitoramento das formas de onda de tensão e corrente em tempo real; relógio e calendário; registro de falhas no fornecimento de energia e de acionamentos da proteção com data e hora em que ocorreram.

Estudos energéticos diferenciam-se das análises econômicas, pois permitem estimar a energia diretamente consumida e/ou indiretamente utilizada em um processo produtivo (SALLA et al. 2009). Em indústrias sucroalcooleiras os índices de eficiência podem ser observados nos trabalhos de Schirmer (2006), que verificou os indicadores de eficiência energética e exergética em duas indústrias do setor sucroalcooleiro, essaltando no setor a necessidade do uso racional da energia como fator de modernização e diversificação.

A análise de indicadores energéticos para avaliação de eficiência nas indústrias tem o objetivo de entender a influência técnico-econômicas no total do consumo final de energia na indústria e individualmente dos subsetores ou filiais. (EICHHAMMER; MANNSBART, 1997).

Para Marques (2006) o desenvolvimento de um diagnóstico energético ou auditoria energética permite determinar quem, quando e como se está consumindo energia. Para Domanski (2011) o setor industrial tem o maior consumo de energia, e faz-se necessário analisar e avaliar as reais situações desse consumo.

Indicadores do uso de energia constituem valores para a realização de diagnósticos energéticos, permitindo verificar o perfil de consumo de uma instalação elétrica e a determinação do potencial de racionalização dessa energia elétrica. Assim para comparar os parâmetros de eficiência energética existentes, verifica-se a alguns conceitos como o Fator de Potência (FP).

O indicador de (FP) é definido como a razão entre a potência ativa e a potência aparente. Ele indica a eficiência no uso da energia, assim um alto fator de potência indica uma eficiência alta e inversamente, um fator de potência baixo indica baixa eficiência energética (CANEPPELE, 2011).

Um triângulo retângulo é freqüentemente utilizado para representar as relações entre potência ativa $(\mathrm{kW})$, potência reativa $(\mathrm{kVAr})$ e potência aparente $(\mathrm{kVA})$, conforme Figura 1.

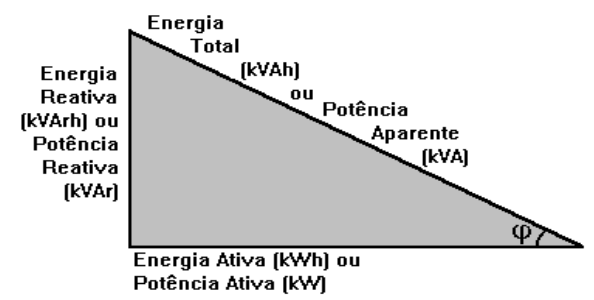

Figura 1: Relação entre energia total, energia ativa e energia reativa

O (FP) é a razão entre a energia elétrica ativa e a raiz quadrada da soma dos quadrados das energias elétricas ativa e reativa, consumidas num mesmo período específico, conforme mostra a Equação 1.

$$
F \quad P\left(\frac{P}{\sqrt{(P)^{2}+(Q)^{2}}}\right)
$$

Em que: 
$\mathrm{FP}=$ valor do fator de potência;

$\mathrm{P}=$ potência ativa, em $[\mathrm{kW}]$;

$\mathrm{Q}=$ potência reativa, em [kVAr].

De acordo com Guelfi (2007), Cremasco (2008) a potência ativa é aquela que produz trabalho útil, expressa em quilowatt $(\mathrm{kW})$. A potência aparente é a potência total absorvida por uma instalação elétrica, usualmente expressa em quilovolt-ampère (kVA) e é obtida da soma geométrica da potência ativa com a potência reativa.

A potência reativa é aquela utilizada para criar o fluxo magnético necessário ao funcionamento dos equipamentos industriais (motores, transformadores, reatores, entre outros) sendo usualmente expressa em quilovolt-ampère-reativo ( $\mathrm{kVAr})$.

Conforme Companhia Energética de São Paulo (COMPANHIA ENERGÉTICA DE SÃO PAULO, 1990), o (FP) quando mantido a índices inferiores a 92\% provoca diversos inconvenientes, entre eles, um acréscimo na conta de energia elétrica.

Os componentes dos sistemas elétricos (geradores, circuitos de transmissão e distribuição, transformadores, instalações internas dos consumidores entre outros) quando operados com excesso de potência reativa, comprometem desnecessariamente a componente ativa da potência total ou aparente. Assim as principais causas do baixo fator de potência são: motores operando a vazio; motores superdimensionados; transformadores operando em vazio ou com pequenas cargas; nível de tensão acima do normal.

O objetivo deste trabalho é analisar os parâmetros de eficiência elétrica para o setor sucroalcooleiro em três empresas da região centro-oeste paulista utilizando-se análise estatística.

\section{MATERIAL E MÉTODOS}

A escolha das usinas, localizadas na região centro-oeste do estado de São Paulo, justifica-se por serem empresas com grande potencial de produção e exportação de açúcar e etanol. Pelo acordo de sigilo para a execução das pesquisas junto às usinas, as usinas serão chamadas de USA, USB e USC com informações técnicas, conforme Tabela 1.

Tabela 1:Valores médios característicos das usinas para safra 2008/2009 para um período de 48 horas.

\begin{tabular}{l|l|l|l}
\hline \multicolumn{1}{c|}{$\begin{array}{c}\text { Descrição } \\
\text { Usinas }\end{array}$} & \multicolumn{1}{c|}{ USA } & \multicolumn{1}{c|}{ USB } & \multicolumn{1}{c}{ USC } \\
\hline $\begin{array}{l}\text { Moagem de cana- } \\
\text { de-açúcar }\end{array}$ & 70.609 ton. & 20.217 ton. & $\begin{array}{l}17237 \\
\text { ton. }\end{array}$ \\
$\begin{array}{l}\text { Produção de açúcar } \\
\text { Produção de etanol }\end{array}$ & 6000 ton. & 1693 ton. & 2400 ton. \\
\hline
\end{tabular}

Fonte: Dados obtidos junto às usinas em estudo.

Em cada usina, foram definidos cinco setores de avaliação:
Setor 1 - Recepção: onde a cana-de-açúcar é pesada, lavada e direcionada aos picadores que trituram os colmos;

Setor 2 - Moagem: a cana-de-açúcar é desfibrada e comprimida por rolos que a submetem a uma pressão de aproximadamente $250 \mathrm{~kg} . \mathrm{cm}-2$ expulsando o caldo do interior das células;

Setor 3 - Caldeiras: o bagaço que sai da moenda com pouco açúcar e aproximadamente $50 \%$ de umidade é transportado para as caldeiras, onde é queimado para gerar vapor, que se destina a todas as necessidades que envolvem o acionamento das máquinas pesadas, geração de energia elétrica e o processo de fabricação de açúcar e etanol;

Setor 4 - Atividades auxiliares/tratamento do caldo: responsável pelo bombeamento de água e iluminação do setor administrativo;

Setor 5 - Destilarias: separar o álcool etílico contido no vinho na proporção de 7 a $10 \%$ dos demais componentes contidos no vinho.

Utilizando cinco equipamentos analisadores de energia Landys+Gyr SAGA modelo 4500, sendo dois deles capazes de medir pontos com corrente máxima de 1000 A e três com corrente máxima de 2000 A e um analisador de energia Landys+Gyr SAGA 4000 com capacidade de medição para corrente máxima de 2000A.

O analisador e registrador SAGA é um dispositivo eletrônico para sistemas elétricos monofásicos ou trifásicos equilibrados ou não. Sua utilização é do tipo portátil em redes de distribuição, para instalação ao tempo ou em cabines primárias, em circuitos diversos de baixa tensão, sem necessidade de desligamento do circuito e atende a portarias do DNAEE e da ANEEL.

Na Figura 2, têm-se o analisador portátil Saga 4500 ligado em rede trifásica junto à entrada de energia na USB.
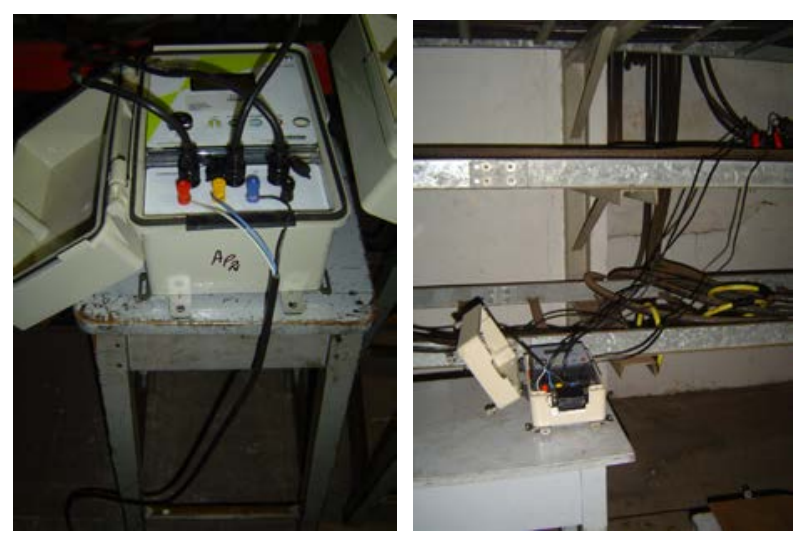

Figura 2: Analisador portátil Saga 4500 ligado em rede trifásica junto à entrada de energia na Usina USB.

Os analisadores de energia foram instalados nos setores de produção em circuitos de alta e baixa tensão no período de três a cinco dias, e foi escolhido um período 
de 48 horas de máxima demanda, para realização dos cálculos. Quando necessário foram realizadas medições simultâneas em mais de um setor ou alimentador para se somarem os resultados.

O analisador foi programado para fazer leituras de dados em intervalos de 15 minutos (padrão resolução 465 da ANEEL). Foram realizadas medições individualizadas para cada setor da produção, envolvendo o processo de produção de açúcar e etanol em função dos diagramas elétricos.

As medições nas três usinas foram realizadas no período de julho a dezembro de 2008, coincidindo com o pico da safra na região. Os parâmetros elétricos foram medidos, com Analisadores de Energia Landys+Gyr SAGA, sendo: tensão em cada fase, em Volts; corrente por fase, em Ampére; potência ativa trifásica (Pt), em kW; potência reativa trifásica $(\mathrm{Qt})$, em kVAr; potência aparente trifásica (St), em kVA; e fator de potência trifásico (FPt) e por fase.

\subsection{Energia consumida na produção de etanol}

Para caracterizar os processos de produção de etanol relacionados aos cincos setores, tanto a demanda quanto o consumo de energia foram calculados considerando o consumo de caldo e quantidade de cana-de-açúcar processada para a produção de etanol (45\%) e do açúcar (55\%), como é usualmente feito nas usinas.

A usina USA possui um setor de atividades auxiliares, que constitui um diferencial na distribuição elétrica, correspondendo o consumo de energia de $50 \%$ destinados à produção de etanol e 50\% destinados a produção de açúcar. Nas usinas USB e USC este setor não é separado dos demais e possuem um setor denominado de tratamento de caldo.

Desse modo, para os cálculos utilizou-se a mesma distribuição conforme as porcentagens de rateio entre as produções de açúcar e etanol para as usinas. Para o cálculo do consumo de energia envolvendo a produção de etanol, foi utilizada a Equação 2 para a usina USA e a Equação 3 para as usinas USB e USC.

$$
\begin{aligned}
& C_{E}=\left(C_{R}+C_{M}+C_{C}\right) \times 0,4+\left(C_{\AA} \times 0,5\right)+C_{D} \\
& C_{E}={ }_{k}\left(C_{R}+C_{M}+C_{C}+C_{T}\right) \times{ }_{C} 0,4+C_{B}
\end{aligned}
$$

Em que:

$C_{E T}=$ Consumo de energia para a produção do etanol, em [kWh];

$C_{R}=$ Consumo de energia do setor de recepção, em [kWh];

$C_{M}=$ Consumo de energia do setor da moagem, em [kWh];

$C_{C}=$ Consumo de energia do setor das caldeiras, em [kWh];
$C_{T C}=$ Consumo de energia do setor de tratamento de caldo, em [kWh];

$C_{A A}=$ Consumo de energia do setor das atividades auxiliares, em [kWh];

$C_{D}=$ Consumo de energia do setor da destilaria, em [kWh];

\subsection{Fator de potência médio para produção de etanol}

Para o cálculo do $F P$ de potência médio para a produção do etanol $F P_{E T}$, foi utilizada a Equação 4 para a usina USA e a Equação 5 para as usinas USB e USC.

Equação 4:

$F P_{E T}=\frac{\left(D_{R} \times F P_{R}+D_{M} \times F P_{M}+D_{C} \times F P_{C}\right) \times 0,45+\left(D_{A A} \times F P_{A A}\right) \times, 05+D_{D} \times F P_{D}}{D_{E T}}$ Equação 5:

$\left(D_{R} \times F P_{R}+D_{M} \times F P_{M}+D_{C} \times F P_{C}+D_{T C} \times F P_{T C}\right) \times 0,45+D_{D} \times F P_{D}$

$$
F P_{E T}=-D_{E T}
$$

Em que:

$\boldsymbol{F} \boldsymbol{P}_{E T}=$ Fator de potência médio para a produção do etanol.

As variáveis analisadas foram: energia consumida (C), fator de potência (FP) e para a análise estatística, adotouse o delineamento de blocos casualizados, em arranjo fatorial $3 \times 5$, composto das três empresas sucroalcoleiras e 5 setores de consumo energético sendo: recepção (1), moagem (2), caldeiras (3), atividades auxiliares/tratamento de caldo (4) e destilaria (5) totalizando 15 tratamentos, sendo cada grupo composto por 192 repetições (48 horas $\times 4$ aferições por hora).

A análise de variância (Anova) foi realizada para comparação dos grupos, com posterior comparação entre as médias pelo teste de Tukey. Para o conjunto de dados considerado, foram verificadas as pressuposições de homocedasticidade e normalidade.

Vale ressaltar que foi estabelecido para todos os testes um nível de significância de $\alpha=0,05$. Para uma descrição adequada das variáveis em relação aos tipos de variáveis nas diferentes usinas e setores, foi realizada uma análise descritiva para apresentação de resultados em valores de médias e desvio padrão, além da criação de gráfico tipo BoxPlot.

\section{RESULTADOS E DISCUSSÃO}

A partir dos dados coletados pelos analisadores de energia foram obtidos os comportamentos das grandezas 
elétricas. Verificam-se os dados da demanda média, fator de potência médio ponderado e fator de potência na produção do etanol.

Tabela 2: Demanda média, fator de potência e fator de potência médio da produção do etanol.

\begin{tabular}{c|c|c|c|c}
\hline Usinas & Setores & $\begin{array}{c}\text { Demand } \\
\text { a média }\end{array}$ & $\mathbf{F P}$ & \multirow{2}{*}{$\mathbf{F P}_{\text {ET }}$} \\
\cline { 3 - 4 } & & {$[\mathbf{k W}]$} & $\begin{array}{c}\text { Médio } \\
\text { ponderado }\end{array}$ & \\
\hline & 1 & 487 & 0,89 & \\
USA & 2 & 1817 & 0,95 & \\
& 3 & 3039 & 0,86 & 0,85 \\
& 4 & 993 & 0,77 & \\
& 5 & 3897 & 0,84 & \\
\hline & 1 & 108 & 0,59 & \\
USB & 2 & 889 & 0,62 & \multirow{2}{*}{0,83} \\
& 3 & 967 & 0,86 & \\
& 4 & 1315 & 0,90 & \\
\hline & 5 & 783 & 0,87 & \\
& 1 & 17 & 0,59 & \\
USC & 2 & 653 & 0,89 & \multirow{2}{*}{0,84} \\
& 3 & 1049 & 0,81 & \\
& 4 & 168 & 0,81 & \\
\hline
\end{tabular}

Em relação ao fator de potência médio de cada setor, observa-se entre as usinas que não há uma preocupação em fazer a correção deste fator para atingir o valor de 0,92 , visto que as usinas não são penalizadas pelas concessionárias por apresentarem estes baixos valores, por gerarem sua própria energia. Com isso, não se tem uso racional e eficiente da energia. Sendo que a usina USA apresentou melhor resultado para o fator médio para produção do etanol de 0,85 , valor este abaixo de 0,92 , recomendado pelas normas vigentes.

Conforme Cagnon (2007) quando o fator de potência é corrigido e elevado para 0,92 ou mais, a empresa passa a utilizar energia da forma mais correta e econômica, uma vez que há redução das perdas de energia e custos de energia elétrica com liberação da capacidade do sistema.

A análise estatística descritiva das informações relativas aos parâmetros elétricos para a energia consumida (C) e (FP) realizada nas usinas pode ser observada na Tabela 3 e Figura 3, sendo a média e demais parâmetros relativos ao período de 48 horas de medições realizadas.

Também na Figura 3 é possível observar outliers na Energia consumida da Empresa 2 e no Fator de potência da Empresa 1, o que possivelmente não ocorreria se o período de aquisição de dados fosse maior do que 48 horas, período este considerado neste estudo.
Tabela 3: Análise descritiva dos dados de (C) e (FP).

\begin{tabular}{|c|c|c|c|c|c|c|c|c|c|}
\hline 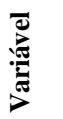 & 营 & : & ถิ & U & $\stackrel{\Xi}{\mathbf{\Xi}}$ & $\bar{\sigma}$ & $\approx$ & $\tilde{\sigma}$ & 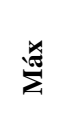 \\
\hline \multirow{3}{*}{$C$} & USA & 2042 & 1290 & $63 \%$ & 377 & 857 & 1991 & 3183 & 4302 \\
\hline & USB & 812 & 404 & $50 \%$ & 37 & 768 & 881 & 1022 & 1501 \\
\hline & USC & 331 & 245 & $74 \%$ & 9 & 167 & 245 & 578 & 715 \\
\hline \multirow{3}{*}{$F P$} & USA & 0,86 & 0,07 & $8 \%$ & 0,58 & 0,84 & 0,86 & 0,90 & 0,97 \\
\hline & USB & 0,77 & 0,14 & $18 \%$ & 0,52 & 0,62 & 0,86 & 0,88 & 0,92 \\
\hline & USC & 0,79 & 0,13 & $16 \%$ & 0,53 & 0,71 & 0,81 & 0,83 & 0,99 \\
\hline
\end{tabular}

Legenda: DP $=$ Desvio padrão, $\mathrm{CV}=$ Coeficiente de Variação, Min = Mínimo, Máx $=$ Máximo, $\mathrm{Q} 1=1 .^{\circ}$ quartil, Q2 $=2 .^{\circ}$ quartil, Q3 $=3 .^{\circ}$ quartil.
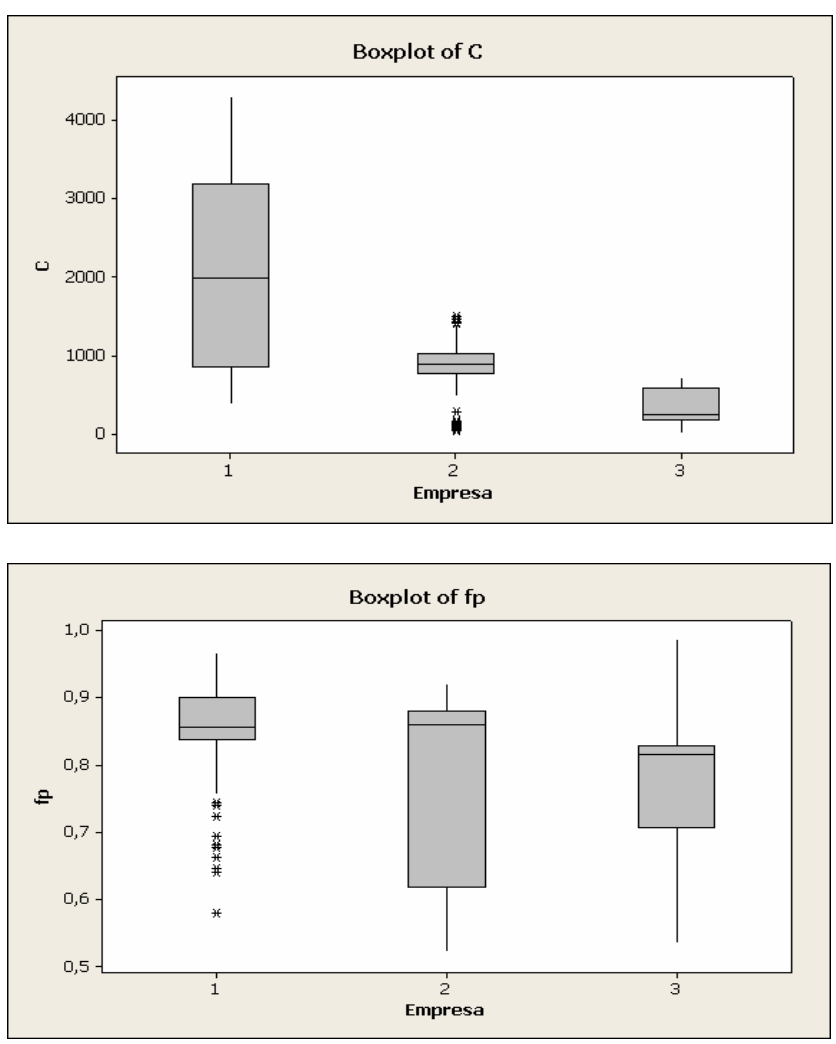

Figura: Boxplot para dados amostrais de (C) e (FP).

De acordo com a Tabela 4, em relação à energia consumida, todos os setores apresentam diferenças significativas. Na comparação setor a setor, a usina USB apresentou diferenças não significativas nos setores 1 e 3 e a usina USC nos setores 1 e 4 . Vale ressaltar que a mesma Tabela apresenta o teste de comparação dos Tratamentos (setores) e Blocos (usinas). 
Tabela 4: Média e desvio padrão das variáveis (C) e (FP), no qual, para cada variável, valores seguidos de mesma letra não diferem ao nível de significância de $5 \%$

\begin{tabular}{|c|c|c|c|c|c|c|c|}
\hline \multirow{2}{*}{ 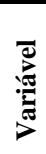 } & \multirow[b]{2}{*}{ ڤัँ } & \multicolumn{6}{|c|}{ USINAS } \\
\hline & & 㟔 & & Uू & & Uू & \\
\hline \multirow[t]{5}{*}{ C } & 1 & $\begin{array}{c}1799 \pm \\
363\end{array}$ & $\mathrm{Aa}$ & $888 \pm 46$ & $\mathrm{Ba}$ & $243 \pm 19$ & $\mathrm{Ca}$ \\
\hline & 2 & $487 \pm 63$ & $\mathrm{Ab}$ & $108 \pm 32$ & $\mathrm{Bb}$ & $17 \pm 4$ & $\mathrm{Cb}$ \\
\hline & 3 & $\begin{array}{c}3039 \pm \\
160\end{array}$ & Ac & $976 \pm 66$ & $\mathrm{Ba}$ & $659 \pm 44$ & Cc \\
\hline & 4 & $993 \pm 259$ & Ad & $\begin{array}{c}1315, \pm \\
115\end{array}$ & Bc & $168 \pm 3$ & $\mathrm{Ca}$ \\
\hline & 5 & $\begin{array}{c}3897 \pm \\
284 \\
\end{array}$ & Ae & $783 \pm 96$ & $\mathrm{Bd}$ & $565 \pm 23$ & Cd \\
\hline$F$ & 1 & $\begin{array}{c}0,94 \pm \\
0,05\end{array}$ & Aa & $\begin{array}{c}0,62 \pm \\
0,02\end{array}$ & $\mathrm{Ba}$ & $0,72 \pm 0,01$ & $\mathrm{Ca}$ \\
\hline \multirow[t]{5}{*}{$P$} & 2 & $\begin{array}{c}0,89 \pm \\
0,01\end{array}$ & $\mathrm{Ab}$ & $\begin{array}{c}0,59 \pm \\
0,02\end{array}$ & $\mathrm{Bb}$ & $0,59 \pm 0,02$ & $\mathrm{Bb}$ \\
\hline & 3 & $0,85 \pm 0$ & Ac & $0,86 \pm 0$ & Ac & $0,81 \pm 0$ & $\mathrm{Bc}$ \\
\hline & 4 & $\begin{array}{c}0,77 \pm \\
0,04\end{array}$ & Ad & $0,9 \pm 0,01$ & $\mathrm{Bd}$ & $0,98 \pm 0$ & $\mathrm{Cd}$ \\
\hline & 5 & $0,84 \pm 0$ & Ae & $\begin{array}{c}0,88 \pm \\
0,02\end{array}$ & Bc & $0,83 \pm 0$ & $\mathrm{Ae}$ \\
\hline & 5 & $0,147 \pm 0$ & $\mathrm{Ab}$ & $\begin{array}{c}0,14 \pm \\
0,001\end{array}$ & Bc & $\begin{array}{c}0,149 \pm \\
0,001\end{array}$ & Ac \\
\hline
\end{tabular}

Legenda: Letras maiúsculas iguais nas linhas representam diferenças não significativas entre as grandezas comparadas nas usinas. Letras minúsculas iguais nas colunas representam diferenças não significativas entre as grandezas comparadas nos setores.

Em relação ao (FP) pode-se observar como comparações principais, a ausência de diferenças significativas para todas usinas, e diferenças significativas entre os setores 3 e 5 para as usinas USA e USC.

\section{CONCLUSÕES}

Analisando o período de safra das medições, pode-se deduzir pelos valores do índice proporcional de consumo de energia na produção do etanol que cada usina estava direcionada para um tipo de produto, sendo: USA para produção de açúcar, usina USB produção de etanol e USC produção de energia.

Conclui-se que uma das principais dificuldades associadas a qualquer programa de conservação de uso final de energia é avaliar e acompanhar o gerenciamento do uso racional de energia em setores industriais complexos.

Considerando as unidades produtoras do setor sucroalcooleiro e que vendem energia elétrica para esse tipo de avaliação é essencial realizar analises, uma vez que os setores analisados são os mais importantes na produção do açúcar e etanol, e analisar e acompanhar esses parâmetros como a energia consumida e Fator de
Potência podem auxiliar com eficiência e maior oferta de energia a ser comercializada.

\section{REFERÊNCIAS}

ANSELMI, R. Eficiência energética exige equipamentos com tecnologia avançada. JORNALCANA, São Paulo, n. 185, maio 2009. Disponível em: $<$ http://www.jornalcana.com.br/pdf/185/\%5Ctecind.pdf> Acesso em: 25 mar. 2011.

CAGNON, J. A. Fator de potência, 2007. Disponível em:http://www.dee.feb.unesp.br/ cagnon/ENERGIA /FATOR\%20DE\%20POTENCIA.pdf. Acesso em: 25 mar.2011

CANEPELLE. F. L. Análise de indicadores de eficiência energética na indústria madeireira através da aplicação da teoria fuzzy. 2011. 207 f. Tese (Energia na Agricultura)- Universidade Estadual de São Paulo, Faculdade de Ciência Agronômicas, Botucatu, 2011.

CREMASCO GABRIEL, C. P. Aplicação da lógica fuzzy para avaliação do Faturamento do consumo de energia elétrica e demanda de uma empresa de avicultura de postura, 2008. 108 f. Tese (Doutor em Agronomia). Faculdade de Ciências Agronômicas da UNESP, Botucatu, 2008. Disponível em: $<$ http://www.fca.unesp.br/pos_graduacao/Teses/PDFs/A rq0287.pdf>. Acesso em: 21 out. 2011

COMPANHIA ENERGÉTICA DE SÃO PAULO. Estrutura tarifária horo-sazonal. São

Paulo, 1990. 17 p.

DOMANSKI. E. L. V. Eficiência energética aplicada aos processos produtivos em uma indústria química. 2011. 89 f. Dissertação (Tecnologia, Engenharia e Modelagem)- Universidade Federal do ABC, Santo André, 2011. Disponível em: $<$ http://pgene.ufabc.edu.br/conteudo/bloco2/publicacoes /Dissertacoes2011/DissertacaoEmerson.pdf>. Acesso em: 10 out.2011

EICHHAMMER, W.; MANNSBART W. Industrial energy efficiency. Indicators for a European crosscountry comparison of energy efficiency in the manufacturing industry. Energy Policy, Amsterdam, v. 25, n. 7/9, p. 759-772, 1997.

FILGUEIRAS, J. E. O.; PINTO, D. F.; RODRIGUES, C. R. B. Sistemas de gerenciamento no combate ao desperdício de energia. In: CONGRESSO BRASILEIRO DE ENERGIA, 11., 2006, Rio de Janeiro. Anais... Rio de Janeiro: COPPE, 2006. p. 1807.

GUELFI, R. Análise da relação entre o faturamento do consumo de energia elétrica e demanda de 
potência ativa e reativa utilizando hiperbolóides de carga e potência. 2007. 138 f. Dissertação (Mestrado em Engenharia Elétrica)-Faculdade de Engenharia, Universidade Estadual Paulista, Ilha Solteira, 2007.

MARQUES, M.; HADDAD, J.; MARTINS, A. R. S. Conservação de energia eficiência

energética de instalações e equipamentos. 3. ed. Itajubá: FUPAI, 2006. 597p.

SALLA, D. A. et al. Avaliação energética da produção de etanol utilizando como matéria-prima a

cana-de-açúcar. Ciência Rural, Santa Maria, v. 39, n. 8, p. 2516-2520, nov. 2009. Disponível em: <www.scielo.br/pdf/cr/2009nahead/a307cr1202.pdf>.

Acesso em: 02 jun. 2010.

SCHIRMER, F. Comparação de indicadores de eficiência energética e exergética em duas indústrias do setor sucroalcooleiro. 2006. 88 f. Dissertação (Engenharia Agrícola)- Universidade Estadual do Oeste do Paraná, Cascavel, 2006. Disponível em: $<$ http://tede.unioeste.br/tede/tde_busca/processaPesquis a.php?listaDetalhes[]=83\&processar=Processar $>$.

Acesso em: 20 jun. 2011.

UNIÃO DA INDÚSTRIA DE CANA-DE-AÇÚCAR. Bioeletricidade vai ter fatia maior na matriz energética. São Paulo, 2011. Disponível em: $<$ http://www.unica.com.br/dadosCotacao/estatistica>. Acesso em: 02 jun. 2011. 